\title{
South-north asymmetry of field-aligned currents in the magnetotail observed by Cluster
}

\author{
J. K. Shi, ${ }^{1}$ Z. W. Cheng, ${ }^{1}$ T. L. Zhang, ${ }^{2}$ M. Dunlop, ${ }^{3}$ Z. X. Liu, ${ }^{1}$ K. Torkar ${ }^{2}$ \\ A. Fazakerley, ${ }^{4}$ E. Lucek, ${ }^{5}$ H. Rème, ${ }^{6}$ I. Dandouras, ${ }^{6}$ A. T. Y. Lui, ${ }^{7}$ Z. Y. Pu, ${ }^{8}$ \\ A. P. Walsh, ${ }^{4}$ M. Volwerk, ${ }^{2}$ A. D. Lahiff, ${ }^{4}$ M. G. G. T. Taylor, ${ }^{9}$ A. Grocott, ${ }^{10}$ \\ L. M. Kistler, ${ }^{11}$ M. Lester, ${ }^{10}$ C. Mouikis, ${ }^{11}$ and C. Shen ${ }^{1}$ \\ Received 11 May 2009; revised 4 February 2010; accepted 4 March 2010; published 29 July 2010.
}

[1] We statistically investigated features of the field-aligned current (FAC) distribution in plasma sheet boundary layers between 17 and $19 R_{E}$ in the magnetotail using the curlometer technique to calculate the current from four-point magnetic field measurements taken in 2001. The results show that the FAC distribution in the plasma sheet boundary layers in the magnetotail has dusk-dawn asymmetry, earthward-tailward (polarity) asymmetry, and north-south asymmetry. The occurrence and polarities of FACs in the Northern Hemisphere are different from those in the Southern Hemisphere. The average density and the standard deviation of the FACs that are most likely to be connected to the Earth are $4.90 \mathrm{nA} \mathrm{m}^{-2}$ and $2.55 \mathrm{nA} \mathrm{m}^{-2}$ in the Northern Hemisphere and $4.21 \mathrm{nA} \mathrm{m}^{-2}$ and $1.80 \mathrm{nA} \mathrm{m}^{-2}$ in the Southern Hemisphere, respectively. For investigating the mechanism of the north-south asymmetry, we mapped the FACs along the field line into the polar region. The footprints of the FACs also show a difference between the Southern and Northern hemispheres (as a function of mapped latitude). These characteristics suggest a north-south asymmetry of the FACs in the magnetosphere. Further investigation is needed to identify the causes of this asymmetry, although the configuration of the magnetosphere, the polar cap boundary, the conductivity in the ionosphere, or the various solar wind-magnetosphere interaction processes all may be contributors. That the FAC densities are different between the hemispheres suggests that an important source of these currents must be a voltage generator.

Citation: Shi, J. K., et al. (2010), South-north asymmetry of field-aligned currents in the magnetotail observed by Cluster, J. Geophys. Res., 115, A07228, doi:10.1029/2009JA014446.

\footnotetext{
${ }^{1}$ State Key Laboratory of Space Weather, Center for Space Science and Applied Research, Chinese Academy of Sciences, Beijing, China.

${ }^{2}$ Space Research Institute, Austrian Academy of Sciences, Graz, Austria.

${ }^{3}$ Space Science and Technology Department, Rutherford Appleton Laboratory, Didcot, UK.

${ }^{4}$ Mullard Space Science Laboratory, University College London, London, UK.

${ }^{5}$ Blackett Laboratory, Space and Atmospheric Physics Group, Imperial College London, London, UK.

${ }^{6}$ Center d'Etude Spatiale des Rayonnements, University of Toulouse, Center National de la Recherche Scientifique, Toulouse, France.

${ }^{7}$ Applied Physics Laboratory, Johns Hopkins University, Laurel, Maryland, USA.

${ }^{8}$ School of Earth and Space Sciences, Peking University, Beijing, China.

${ }^{9}$ European Space Research and Technology Centre, European Space Agency, Noordwijk, Netherlands.

${ }^{10}$ Department of Physics and Astronomy, University of Leicester, Leicester, UK.

${ }^{11}$ Department of Physics, University of New Hampshire, Durham, New Hampshire, USA.
}

Copyright 2010 by the American Geophysical Union. 0148-0227/10/2009JA014446

\section{Introduction}

[2] Field-aligned currents (FACs) exist in many geospace regions, such as the polar ionosphere and the magnetotail. They have an important role for the transfer of momentum and energy between the solar wind, the magnetosphere, and the ionosphere, and for dynamic processes in the magnetosphere-ionosphere coupling. In the early 1960s, FACs were detected for the first time from satellites [Zmuda et al., 1966; Cummings and Desler, 1967]. The properties of the FACs have been examined, and some statistical characteristics of the FACs have been studied [Fujimoto et al., 2001]. Iijima and Potemra [1978] made a statistic of the characteristics of large-scale FACs above the ionosphere by using magnetic field data by the TRIAD satellite at $\sim 800 \mathrm{~km}$ altitude. They investigated region $1(\mathrm{R} 1)$ and region 2 (R2) currents in the polar region. Elphic et al. [1985] used the magnetometer data from ISEE 1 and 2 to analyze 189 plasma sheet boundary layer (PSBL) crossings and found that the distribution of the current polarity for small-scale currents at the poleward boundary is opposite to that of the R1 current system. Ohtani et al. [1988] analyzed the FAC in the PSBL, 
also using magnetometer data from the ISEE 1 and 2, and found that the polarity of the large-scale R1 current system is consistent with that of the lower latitude FACs in the PSBLs in the magnetotail; indeed, they found evidence for two possible sources of the large-scale currents located $<15 R_{E}$ and $>15 R_{E}$. Ueno et al. [2002] statistically studied the outermost FACs observed by the Geotail spacecraft while crossing the PSBL inside $X_{\mathrm{GSM}}=-40 R_{E}$. Their results showed a dawn-dusk asymmetry of the polarity of the outmost FACs on the earthward side of the reconnection site. Ohtani et al. [2005] studied seasonal variations of large-scale FAC systems using the magnetic field measurements from the DMSP F7 and F12-F15 satellites. Their focus was placed on the latitudinal extent at the demarcation between the R2 and R1 currents and the intensities of these currents. The results showed that the dayside FAC moves poleward and equatorward in the summer and winter hemispheres, respectively, and the nightside FAC has the opposite seasonal dependence. Christiansen et al. [2002] studied the seasonal dependence of the dynamic properties of the dayside and nightside FAC systems over the Northern and Southern hemispheres, as inferred from Ørsted and Magsat observations over polar region. Their results showed that the dawn-dusk distance between R1/R2 currents exhibits little seasonal dependence in both polar caps. In all the research mentioned above, the magnetic field data from a single satellite were used to estimate the FAC. This calculation may be ambiguous because the time and space variations can not be fully distinguished by single-point measurement.

[3] The Cluster satellites lie in an inertial, polar, $4 \times 19.6 R_{E}$ orbit and give multipoint measurements of magnetic field data [Balogh et al., 2001], providing with us a good opportunity to calculate electric current with the so-called curlometer technique [Robert and Dunlop, 1998; Dunlop et al., 2002]. Vallat et al. [2005] studied the current density in the ring current region using simultaneous multispacecraft Cluster FGM magnetometer data with the curlometer technique. Recently, Alexeev et al. [2005] presented Cluster observations of parallel electron currents only using 3-D, $4 \mathrm{~s}$ resolution data of the PEACE electron spectrometer in the plasma sheet during periods of reconnection. Draper et al. [2005] also reported Cluster magnetotail observations of a tailward-traveling plasmoid at the substorm expansion phase onset and FACs in the PSBL associated with periods of reconnection. Snekvik et al. [2007] also have used the Cluster data and studied a FAC at the dawn flank of a burst bulk flow in the outer central plasma sheet, close to the midnight sector in the Southern Hemisphere. Forsyth et al. [2008] studied tail current systems associated with bursty bulk flows and auroral streamers during a period of multiple substorms using Cluster data. Using the curlometer technique they determined that the current density of the FACs in the bursty bulk flow have been $\sim 5 \mathrm{nA} \mathrm{km}^{-2}$. So far, no statistical result of the FACs in PSBLs observed by Cluster have been reported.

[4] In this paper, we use the Cluster FGM 4-point magnetic field data measured in the PSBLs in the magnetotail with $X_{\mathrm{GSM}}$ of $\sim 17-19 R_{E}$ in 2001 to study the features of the FAC statistically [Asano et al., 2005]. We find, as our main results, that the FACs have asymmetries in several aspects: dawn-dusk asymmetry of occurrence, asymmetry of polarity (these are consistent with that found by Geotail spacecraft inside $40 R_{E}$ [Ueno et al., 2002]), and, in particular, south-north asymmetry.

\section{Selection of Cases}

[5] The data used for this study are $4 \mathrm{~s}$ average magnetic field data from the FGM instrument on board the four Cluster satellites, taken in 2001 from the middle of July to the end of October. In this period the Cluster array crosses the plasma sheet and its two boundary layers around apogee at $\sim 3$ hours of local time around midnight. Figure 1a shows a cartoon of the PSBLs in the magnetotail and the Cluster orbit. In Figure 1a, the two gray areas schematically show the PSBLs where the FAC cases were selected for this study according to the criteria described below. The ellipse represents the Cluster orbit. The letters "N" and "S" show the north and south PSBL, respectively.

[6] As mentioned above, the FAC cases occur in two PSBLs in the Northern and the Southern Hemisphere, respectively. In this study, we only selected those intervals of FACs located in the PSBLs, identified by low $B_{z}$ field orientations. In addition, we only chose the Cluster orbits that cross the plasma sheet within $Y_{\mathrm{GSM}}$ from $-15 R_{E}$ to $15 R_{E}$ ( $\sim 3$ hours LT around midnight) to avoid the low-latitude boundary region on the flanks. To select a FAC case, the following conditions must be met. (1) $B_{X Y}=$ $\sqrt{B_{X}^{2}+B_{Y}^{2}}>15 \mathrm{nT}$ to exclude the cross-tail current region. It is known that the plasma sheet is always flapping in the $Z$ direction, and it is difficult to identify the PSBL using a fixed-coordinate system as reference. Ideally, one shall use $\beta$ to define the PSBL region. However, since in this paper we limited our data to FGM only, we apply the condition $B_{X Y}>15 \mathrm{nT}$ as a proxy. (2) The density of the FAC exceeds $3 \mathrm{pT} / \mathrm{km}$. (It is $\sim 2.4 \mathrm{nA} \mathrm{m}^{-2}$ because $1.2566 \mathrm{pT} / \mathrm{km}=1 \mathrm{nA}$ $\mathrm{m}^{-2}$.) This ensures that the current background noise and the errors resulting from the current calculation using the tetrahedron approximation are low. (3) The interval between consecutive cases is more than $10 \mathrm{~min}$. Figure $1 \mathrm{~b}$ shows an example of the FAC cases selected in this paper according to the $B_{\mathrm{xy}}, \mathrm{FAC}$ density, and chosen interval. In Figure 1b, all cases A, B, C, and D lie in the PSBLs because $B_{\mathrm{xy}}>15 \mathrm{nT}$, but case $C$ should not be selected because the current density is less than $3 \mathrm{pT} / \mathrm{km}$ and the interval of the case $C$ and $D$ is less than 10 min. Cases A, B, and D were selected because they meet all three conditions mentioned above.

[7] As we know, the current density and the magnetic vector are related through Ampere's law:

$$
\mu_{0} \int_{A} \vec{J} \cdot d s=\int_{C} \vec{B} \cdot d l
$$

Here, $\vec{J}$ is the current, $\vec{B}$ is the magnetic field, and $\mu_{0}$ is the magnetic constant. We calculate the current using the curlometer technique that is based on Ampere's law, but estimate the integrals numerically from the spatially differenced data. Considering that the separation of each two Cluster satellites near apogee is $\sim 2000 \mathrm{~km}$ in the 2001 phase and the configuration of the magnetic field is in the magnetotail PSBL, the curlometer technique [Dunlop et al., 1988, 2002; Robert and Dunlop, 1998] is accurate enough to identify the 
a

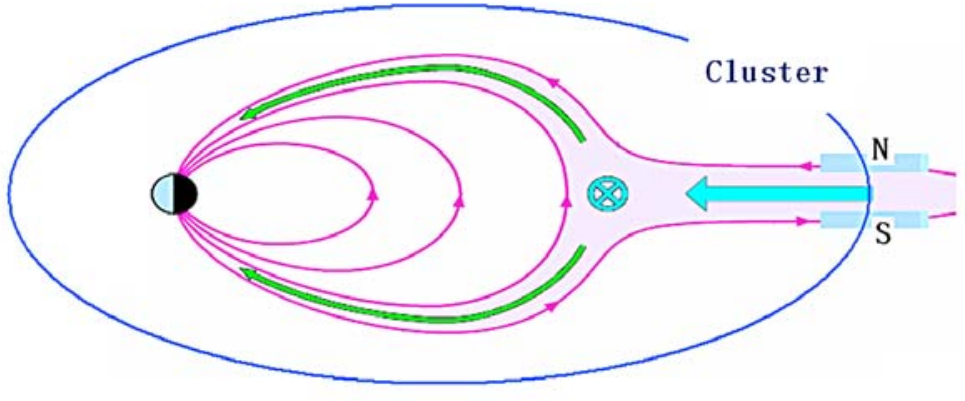

b
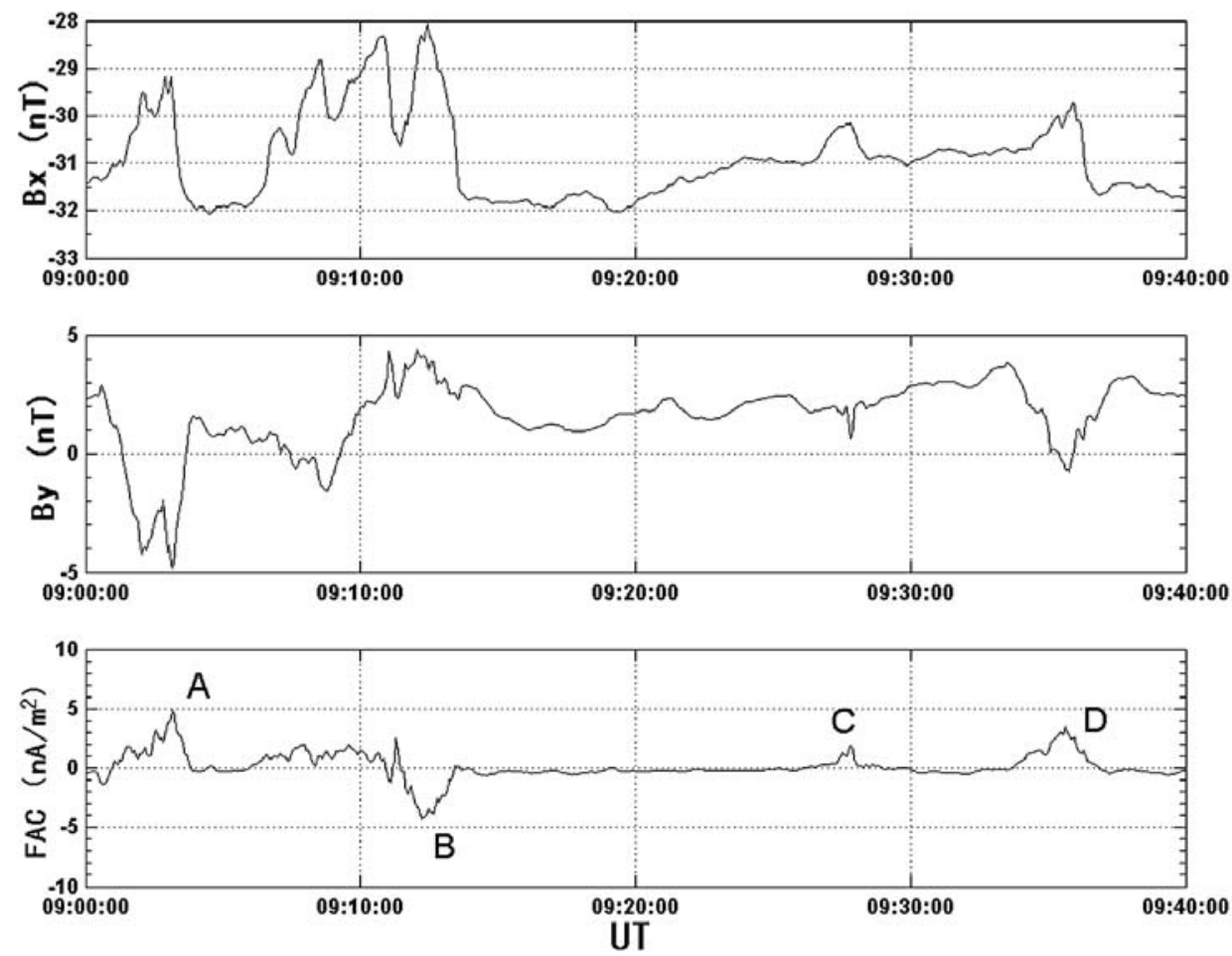

Figure 1. (a) Cartoon of the PSBLs in the magnetotail and the Cluster orbit. The two gray areas show the PSBLs within which the FACs occurred. The letters " $N$ " and "S" show the north and south PSBL, respectively. The ellipse represents the Cluster orbit. (b) Selection procedure for the FAC cases made according to Bxy, FAC density, and interval. All cases A, B, C, and D lie in the PSBLs because Bxy > $15 \mathrm{nT}$, but case $\mathrm{C}$ should not be selected because the current density is less than $3 \mathrm{pT} / \mathrm{km}$ (or $2.4 \mathrm{nAm}^{-2}$ ) and the interval of the case $C$ and $D$ is less than $10 \mathrm{~min}$.

FACs in this study. To ensure that the data quality is sufficient, we choose intervals for which

$$
|\nabla \cdot \vec{B}| /|\nabla \times \vec{B}|<<1
$$

It should be noted that in 2001, between July and late October, the separation between the satellites near apogee and the spacecraft configuration were fairly regular. The relative spacecraft separation scale compared with the spatial scale of the current density in the magnetotail PSBL is therefore favorable for application of the curlometer. We take the ratio in equation (2) to be less than 0.3 to anticipate sufficient accuracy in the analysis of the FAC properties. Typically, however, the curlometer gives estimates of the FAC density with errors of $\sim 12 \%$ for the set of cases chosen here (Figure 1b (bottom), for example, shows that current is approximately zero $\left(<0.5 \mathrm{nA} \mathrm{m}^{-2}\right)$, except for the FAC cases with an average density of $\sim 4.2-4.9 \mathrm{nA} \mathrm{m}^{-2}$ ). We would like to emphasize that the calculation using four spacecraft data gives an unambiguous estimate of the current within this accuracy.

\section{Statistical Results}

[8] According to the prescription in section 2, 172 cases were selected to statistically study the properties of the FACs. The left-hand panel of Figure 2 shows the mean $B_{x}-B_{y}$ components for all the selected cases and shows that all the cases have $B_{X}>15 \mathrm{nT}$, suggesting that all the selected FAC cases took place in the PSBLs. We also used the plasma $\beta$ to determine the PSBLs $(1>\beta>0.01$ for PSBL [Ueno et al., 2002]) and obtained the same result. It is also apparent that the spread of $B_{y}$ in the Northern Hemisphere is similar to that in the Southern Hemisphere but with 

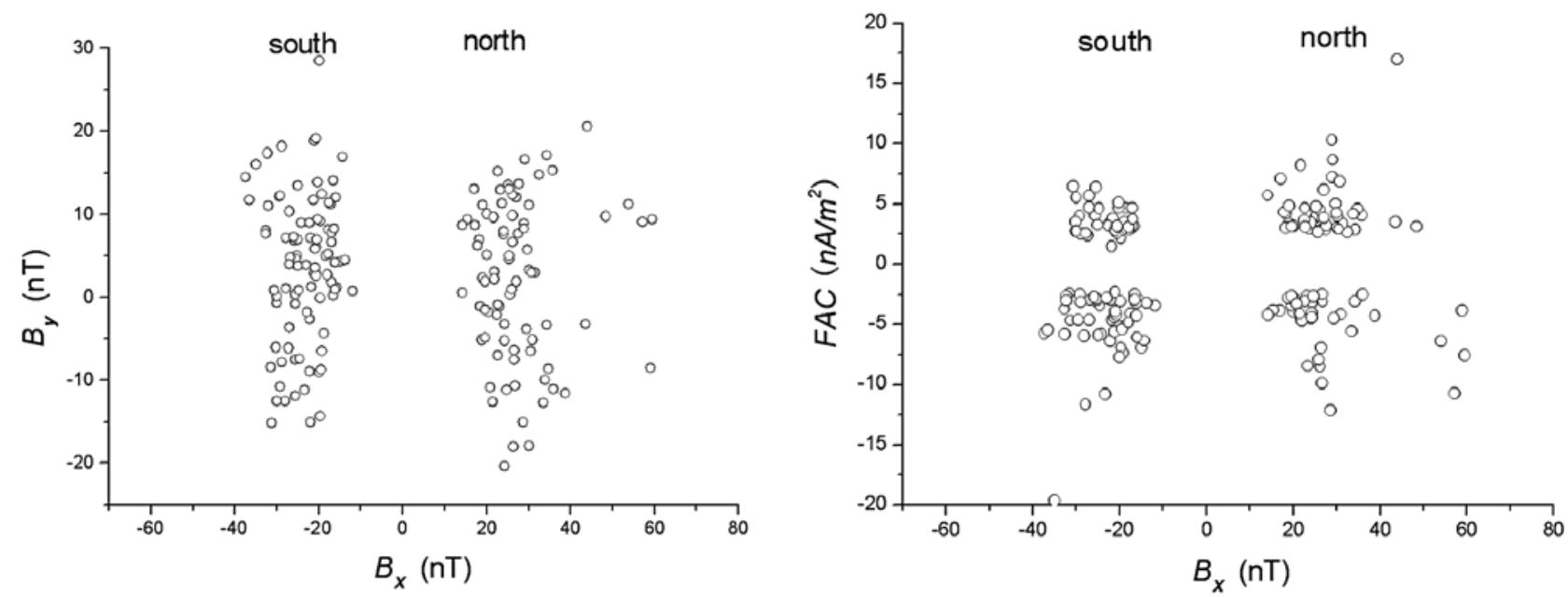

Figure 2. (left) FAC distribution on the $B x-B y$ plane that shows the FAC cases were only selected in the plasma sheet boundary layers and (right) the calculated FAC density distribution for all the cases that shows FAC have a larger range of density values in the north hemisphere.

a larger spread (in the Northern Hemisphere). In Figure 2 (right) we show the distribution of calculated FACs for all the cases. At first glance, this shows a fairly even spread of values both north and south, and for tailward and earthward currents, although again with a larger range of density values in the Northern Hemisphere. We discuss the details of this distribution in Figures 5 and 6.

[9] Figure 3 shows the position and the electric current density of all of the FAC cases projected into the $X-Y$ plane, in the GSM coordinate system. In Figure 3 (left), each arrow represents a FAC case, the starting point of the arrow is at the location of the case (shown as a scatter plot in Figure 3 (right) for clarity), the arrow direction represents the FAC direction, and the arrow length represents the FAC magni- tude of the current density in units of $\mathrm{nA} \mathrm{m}^{-2}$. The righthand panel in Figure 3 shows only the polarity of the FACs (earthward or tailward directed). From Figure 3 we can see that the FACs are fairly evenly distributed over a range of dawn-dusk local times, with only a few cases located at closer radial distances, and that some currents are earthward and some are tailward. The FAC polarity distribution on the dawn side is not the same as that on the dusk side, which also suggests a dusk-dawn asymmetry of the FAC distribution.

[10] To clarify the dawn-dusk distribution, Figure 4a shows the observed FAC distribution versus $Y_{\mathrm{GSM}}$, In Figure $4 \mathrm{a}$, the gray and the black in each block mark the earthward and tailward FACs, respectively. The height of
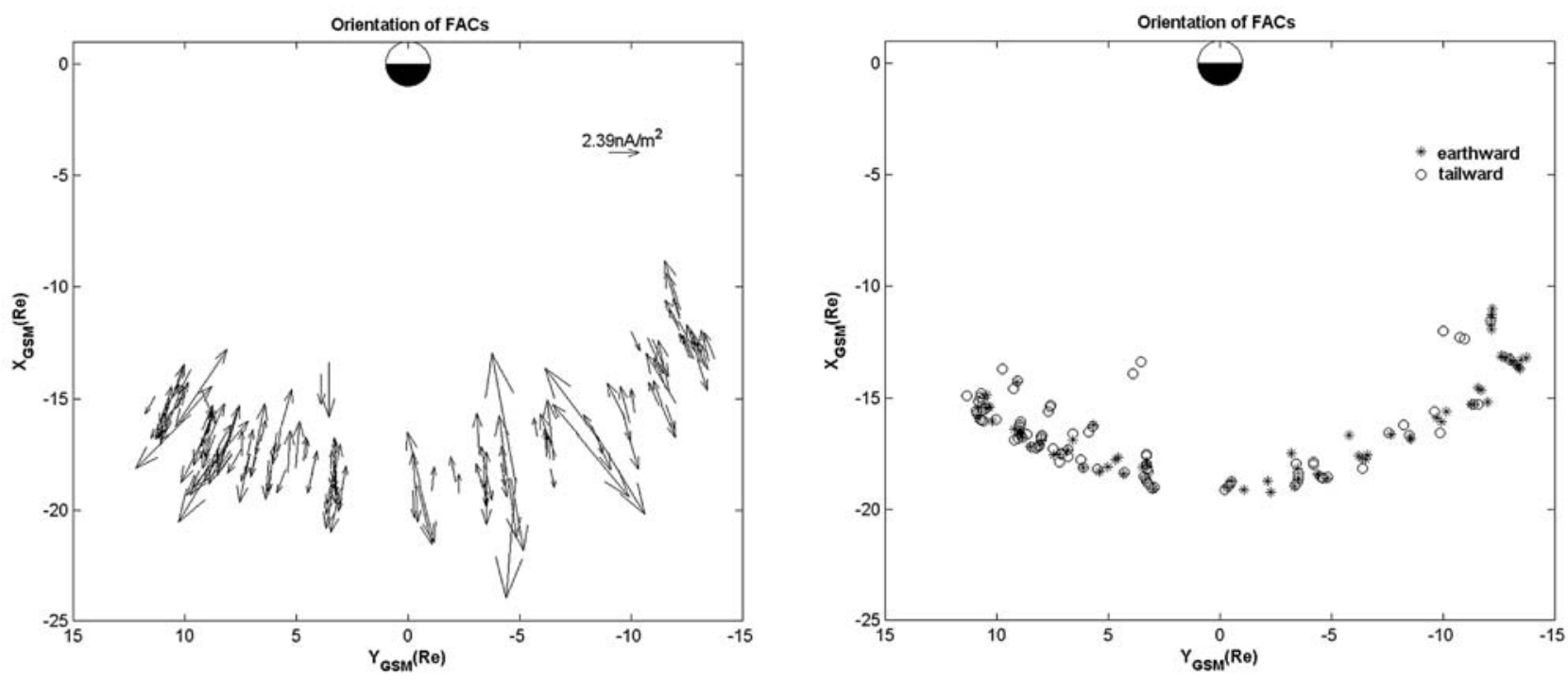

Figure 3. FAC cases on the $X-Y$ plane in the GSM system. (left) The starting point of the arrow is the location of each FAC case and the length of the arrow represents the FAC density, while the arrow direction represents the FAC direction. (right) The FAC location only, the circle shows the tailward FAC, and the cross shows the earthward FAC. This figure shows the FACs' dawn-dusk asymmetry and earthwardtailward asymmetry. 

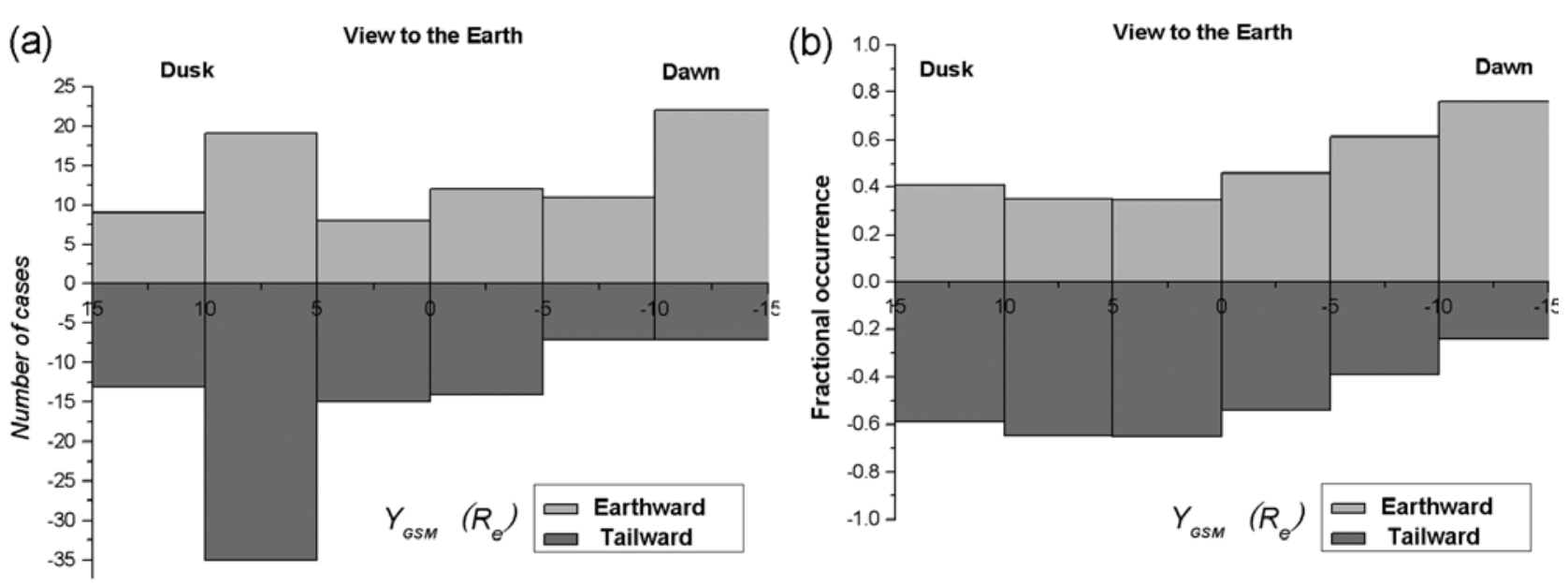

Figure 4. FAC distribution versus $Y_{\mathrm{GSM}}$; gray and black mark the earthward and tailward FACs, respectively. (a) FAC cases number distribution and (b) FAC fractional occurrence.

each block presents the number of observed cases. We can clearly see the FAC distribution in Figure 4a. For the earthward FAC, there are two peak occurrences: one is on the dusk side in the region of $Y_{\mathrm{GSM}}$ from $5 R_{E}$ to $10 R_{E}$ and the other is on the dawn side in the region of $Y_{\mathrm{GSM}}$ from $-15 R_{E}$ to $-10 R_{E}$. These two highest occurrences are nearly identical, but in other regions of $Y_{\mathrm{GSM}}$ both on dusk and dawn side, the occurrences are different. For the tailward FAC, the occurrence is larger and takes place predominantly on the dusk side in the region of $Y_{\mathrm{GSM}}$ from $5 R_{E}$ to $10 R_{E}$. On the dawn side, the occurrences in each region are small. Therefore, we can conclude with our statistical study that both the earthward and tailward FAC distributions are not the same on the dawn side magnetotail and dusk side magnetotail. Therefore, we can say that the FACs have a dusk-dawn asymmetry of occurrence in the PSBLs in the magnetotail.

[11] Figure $4 \mathrm{~b}$ shows the fractional occurrence of the FACs versus $Y_{\mathrm{GSM}}$. In Figure $4 \mathrm{~b}$, the gray presents the earthward FAC and the black presents the tailward FAC in each block. In order to compare the fractional occurrence between the earthward and the tailward FAC distribution more clearly, the height in each block (from bottom of the black to top of the gray) at each $Y$ position is normalized as unit 1, i.e., the height of the gray part of the block (earthward FAC's fractional occurrence) added to the height of the black part of the block (tailward FAC's fractional occurrence) just equal 1 in each block. For example, in the block of $Y_{\mathrm{GSM}}$ from $10 R_{E}$ to $15 R_{E}$, the height of the gray part is 0.42 and the height of the black part is 0.58 . This means that the fractional occurrence of the earthward FAC is 0.42 and the fractional occurrence of the tailward FAC is 0.58 . We can then see more clearly from Figure $4 \mathrm{~b}$ that on the dusk side, the occurrence of the tailward FAC is higher than that of the earthward FAC. On the dawn side, in the region of $Y_{\mathrm{GSM}}=0$ to $-5 R_{E}$, the occurrence of the tailward FAC is slightly higher than that of the earthward. In the region of $Y_{\mathrm{GSM}}=-5 R_{E}$ to $-15 R_{E}$, however, the occurrence of the tailward FAC is lower than that of the earthward. Therefore, we can say that the FACs have polarity asym- metry in the PSBLs in the magnetotail, and the polarity asymmetry is more pronounced on the dawn side.

[12] This dawn-dusk asymmetry of the polarity of FACs in the region of 17-19 $R_{E}$ in the magnetotail shown in this paper is consistent with that expected for the large-scale R1 current system, as found by other work inside $40 R_{E}$ in the magnetotail [Ueno et al., 2002]. In this study, we also investigate the earthward-tailward asymmetry in the Northern and Southern Hemispheres separately. The positions of FACs in the Cluster orbit at $17-19 R_{E}$ in the magnetotail are likely to connect both to the inner R1 current system and to currents at the poleward boundary. It is also possible that, in the event of tail reconnection, a neutral line (NENL) may form either earthward or tailward of the Cluster location. To consider the R1 current system more directly, therefore, we use the sign of $B_{z}$ to filter out any reconnection-driven currents, which arise from a NENL located earthward of the spacecraft, i.e., those lying on field lines with negative $B_{z}$, in general, will not connect to the Earth. Note here that seasonal dependence of the Cluster orbit implies that all cases here correspond toward dipole tilt angles, so that $B_{z}$ is likely to be positive for field lines connecting to the Earth. There are 146 cases with condition $B_{z}>0$ selected for the analysis. Figure 5 illustrates the distribution of all the 146 FAC cases in the Northern Hemisphere (three panels on the left hand) and Southern Hemisphere (three panels on the right hand), respectively. In Figure 5, for both left and right sides, the top two panels show the FAC location on the $X-Y$ plane in the GSM system, and Figure 5 (middle) shows the projected current density in the same format as for Figure 3. Figure 5 (bottom) shows the FAC's fractional occurrence versus $Y_{\mathrm{GSM}}$, corresponding to the FAC cases in the top panels, the gray and the black mark the earthward and tailward FACs, respectively, as for the right-hand side of Figure 4.

[13] From the top two panels in Figure 5, we can clearly see that the distributions of FAC locations are different between the two hemispheres. The distribution of FAC locations is more spread on the dusk side of the Northern Hemisphere and the dawn side of the Southern Hemisphere. In Figure 5 (middle), it can be seen that there are also 

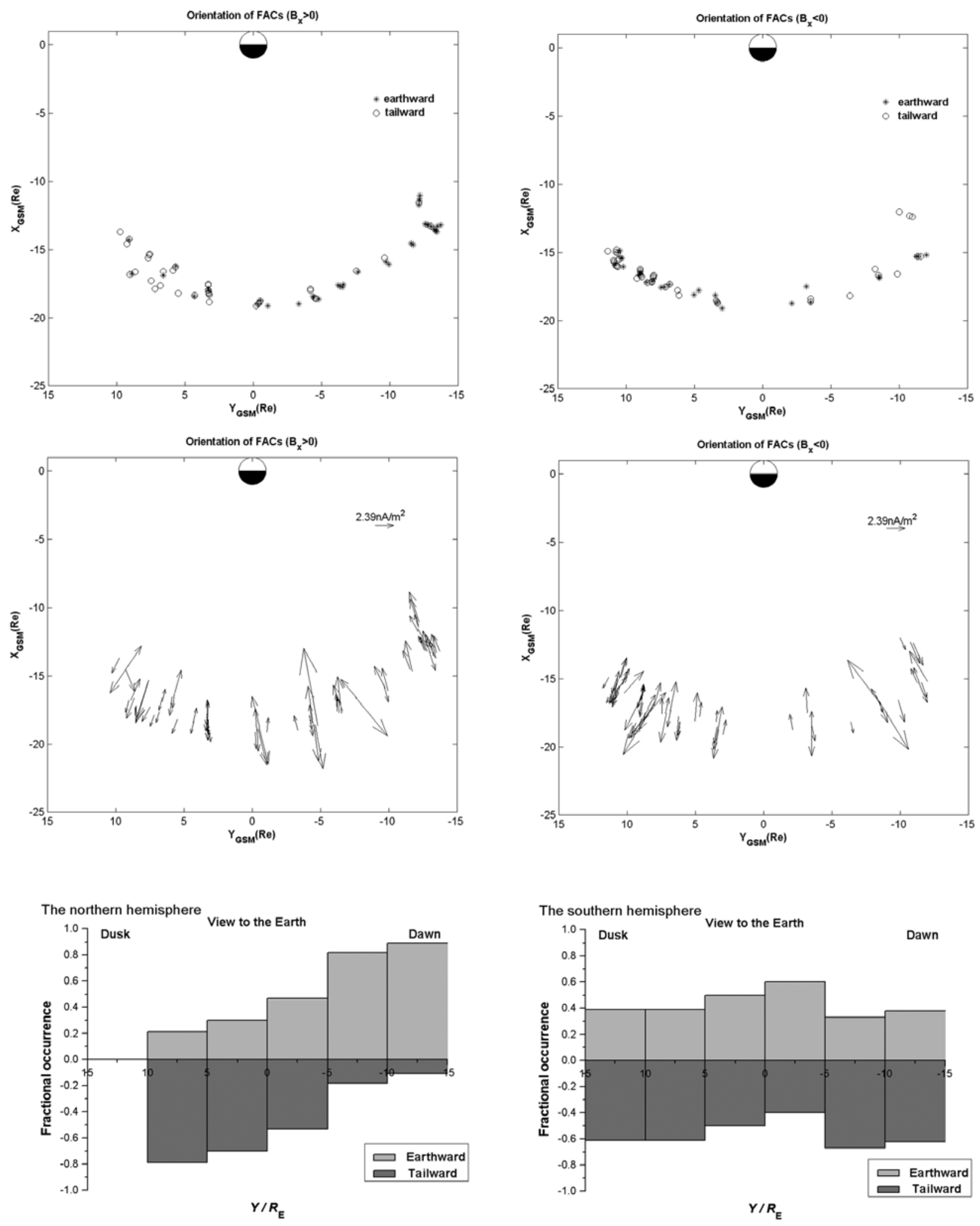

Figure 5. The FAC cases distribution (exclusive the cases with the condition $B_{z}<0$ ) in the (left) Northern Hemisphere and in the (right) Southern Hemisphere, respectively. On each side, the upper two panels show the FAC location on the $X-Y$ plane in the GSM system with the circle to mark the tailward FAC and the star to mark the earthward FAC. Figure 5 (middle) shows the FAC density (marked by arrow length) and polarity (marked by arrow head) correspondence to the FAC cases in the upper panels. Figure 5 (bottom) shows the FACs fractional occurrence versus $Y_{\mathrm{GSM}}$ correspondence to the FAC cases in the upper panels, the gray and the black mark the earthward and tailward FACs, respectively. 
Table 1. The FAC Average Density and Standard Deviation

\begin{tabular}{|c|c|c|c|c|}
\hline & \multicolumn{2}{|c|}{$\begin{array}{c}\text { Northern } \\
\text { Hemisphere }\end{array}$} & \multicolumn{2}{|c|}{$\begin{array}{c}\text { Southern } \\
\text { Hemisphere }\end{array}$} \\
\hline & Earthward & Tailward & Earthward & Tailward \\
\hline Average density $\left(\mathrm{nA} \mathrm{m}^{-2}\right)$ & \multicolumn{2}{|c|}{4.90} & \multicolumn{2}{|c|}{4.21} \\
\hline \multirow[t]{2}{*}{ Standard deviation $\left(\mathrm{nA} \mathrm{m}^{-2}\right)$} & \multicolumn{2}{|c|}{2.55} & \multicolumn{2}{|c|}{1.80} \\
\hline & 2.62 & 2.47 & 1.17 & 2.06 \\
\hline
\end{tabular}

differences in the FAC density distribution and polarity distribution between the two hemispheres. The earthwardtailward polarities (see distributions in the Figure 5 (middle)) show a strong asymmetry from dawn to dusk in the Northern Hemisphere, while this is not so pronounced in the Southern Hemisphere. It is also clear that, while the number of cases is relatively evenly spread in the Northern Hemisphere, the occurrence of cases in the Southern Hemisphere appears to increase from dawn to dusk. Figure 5 (bottom) shows this result statistically. We can see that in the Northern Hemisphere, from $Y=10$ to $15 R_{E}$, there were no FAC measured, which is much different from the situation in the Southern Hemisphere. Also, the dawn-dusk asymmetry both for earthward and tailward FACs in the two hemispheres are not the same. The polarity (earthward or tailward) asymmetry is different in the two hemispheres both on the dawn side and the dusk side. The polarity asymmetry in the northern hemisphere is clearly much stronger than that in the southern hemisphere, which shows only a weak trend.

[14] Supporting this plot, Table 1 shows the average densities and standard deviations of the FACs with condition $B_{z}>0$ (the 146 cases as mentioned above) for both polarities (earthward and tailward) in the different hemispheres. We can see from Table 1 that the average density of all FACs is $4.90 \mathrm{nA} \mathrm{m}^{-2}$ in the Northern Hemisphere and $4.21 \mathrm{nA} \mathrm{m}^{-2}$ in the Southern Hemisphere, and the standard deviation is $2.55 \mathrm{nA} \mathrm{m}^{-2}$ in the Northern Hemisphere and $1.80 \mathrm{nA} \mathrm{m}^{-2}$ in the Southern Hemisphere, respectively. Both the average density of the FACs and its standard deviation in the Northern Hemisphere has a value different from that in the Southern Hemisphere. In addition to this analysis, we have performed Welch's test for the FAC average density in the two hemispheres using the statistical values provided in Tables 1 and 2. The test result shows that, with a test standard $\alpha=0.10$, i.e., $90 \%$ probability, we can conclude that the FACs have different average densities in the Northern and Southern Hemispheres. Also, we can say from Table 1 that both for earthward and tailward FACs, the average density and its standard deviation in the Northern Hemisphere are higher than those in the Southern Hemisphere. Indeed, in the right panel of Figure 2, we can see that the FACs have a larger range of density values in the Northern Hemisphere. That is why the average current density is higher in the Northern than in the Southern Hemisphere.

[15] Table 2 gives the distribution of FAC cases with condition $B_{z}>0$ (the 146 cases as mentioned above) for each sector, polarity, and hemisphere. From Table 2, we can see that, on the dawn side, there were 44 cases taken in the Northern Hemisphere and 19 cases taken in the Southern Hemisphere (for both earthward and tailward currents). We also can see from Table 2 that, on the dusk side, there were 29 cases taken in the Northern Hemisphere and 54 cases taken in the Southern Hemisphere. Thus, there is a larger imbalance in the occurrence of cases in the Southern Hemisphere, with the majority of cases occurring on the dusk side in the south (as seen on the plots in Figure 5). Moreover, for each of the earthward and tailward current cases, either on the dawn side or dusk side, the occurrence of the FACs is different, although the asymmetry is more pronounced for the earthward currents. Also, in the Northern Hemisphere, we see that the dominance of earthward FACs on the dawn side, and tailward FACs on the dusk side, is much more pronounced than in the Southern Hemisphere. The variance in the distribution therefore confirms that the polarity trends are statistically significant. These further show FACs asymmetry in the Northern and Southern Hemispheres.

[16] In summary, we have found that in the Northern Hemisphere the number of the FACs cases is relatively evenly distributed in LT, but that the earthward-tailward polarity has a more pronounced dawn-dusk asymmetry. In the Southern Hemisphere, the occurrence is progressively higher with decreasing LT (from dawn to dusk), but the earthward-tailward asymmetry is much less pronounced.

\section{Discussion in the Polar Ionosphere}

[17] In this study, we also used the T-96 geomagnetic model [Tsyganenko and Stern, 1996] to map all FACs along the magnetic field lines from the FAC location to the polar region to more clearly identify the latitude locations, in particular, near the polar cap boundary. The resulting mapped distribution also showed a difference between the Northern Hemisphere and the Southern Hemisphere. For example, Figure 6a shows the footprints in the polar region in the two hemispheres. In Figure 6a, the left panel is for the footprints of FACs in the Northern Hemisphere and the right panel is for those in the Southern Hemisphere. The stars mark the tailward FAC and the circles mark the earthward. Since the Cluster orbit crosses the PSBL at $\sim 17-19 R_{E}$ radial distance, it is likely that both open- and closed-field lines are sampled mapping to the whole region 1 and polar cap boundary current system in the ionosphere. Here, we again use the 146 cases as above to do the analysis, which have filtered out any reconnection-driven currents arising from a NENL located earthward of the spacecraft, i.e., those lying on field lines not connected to the Earth. Since the cases all occur toward dipole tilt angles, it is likely that any cases for which $B_{z}$ is negative will correspond to disconnected field lines, tailward of an $x$-line.

[18] From Figure 6a we can see that distributions of the FAC footprints are different in the two hemispheres. First, the FAC distribution is from the longitude of 2030 magnetic local time (MLT) to 0415 MLT in the Northern Hemisphere and is from the longitude of 1930 MLT to 0415 MLT in the

Table 2. Statistics of the FAC Case Number

\begin{tabular}{lcclccc}
\hline & \multicolumn{2}{c}{ Dawn Side } & & \multicolumn{2}{c}{ Dusk Side } \\
\cline { 2 - 3 } \cline { 5 - 6 } \cline { 5 - 6 } & Earthward & Tailward & & Earthward & Tailward \\
\hline Northern Hemisphere & 32 & 12 & & 7 & 22 \\
Southern Hemisphere & 8 & 11 & & 22 & 32 \\
\hline
\end{tabular}


a

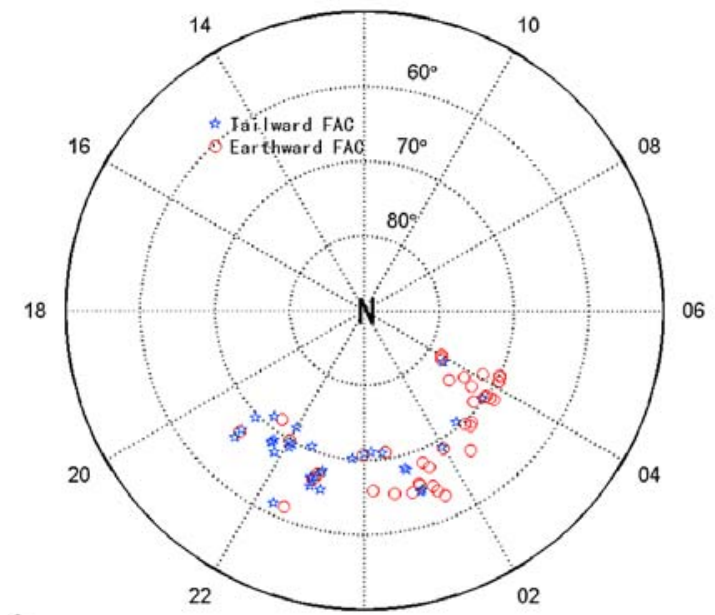

b

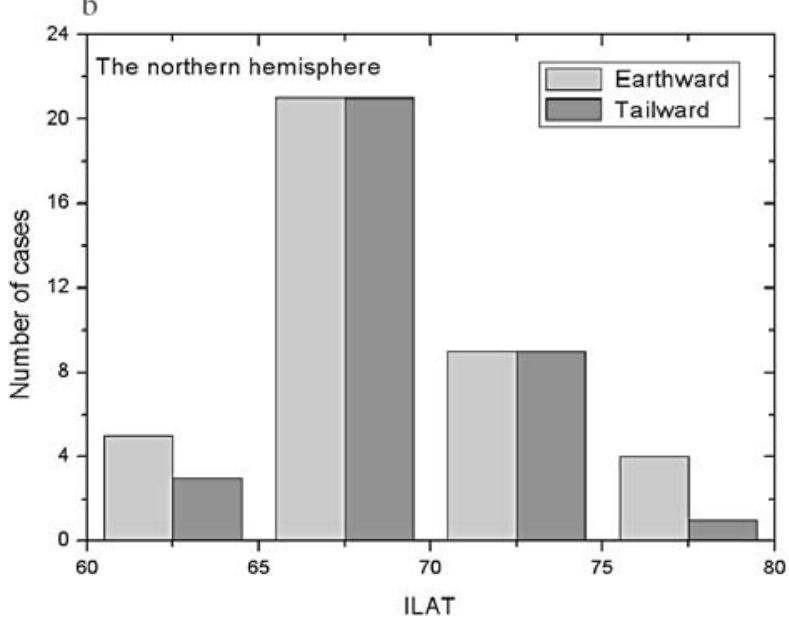

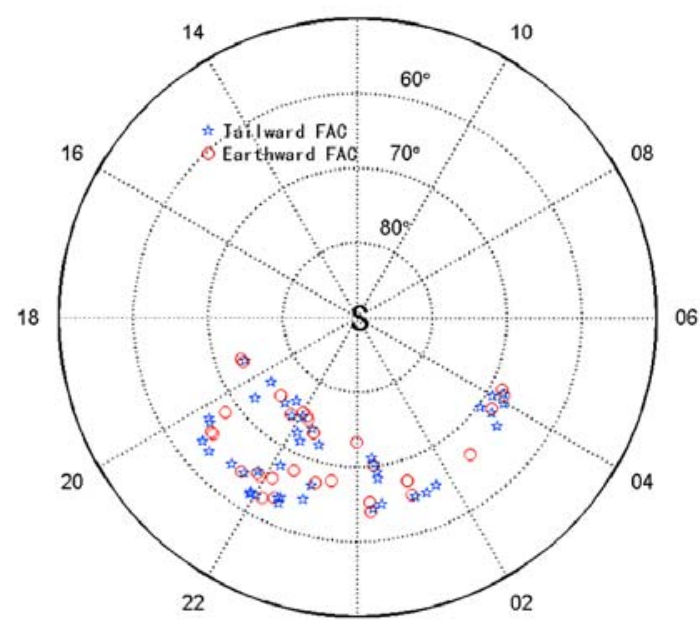

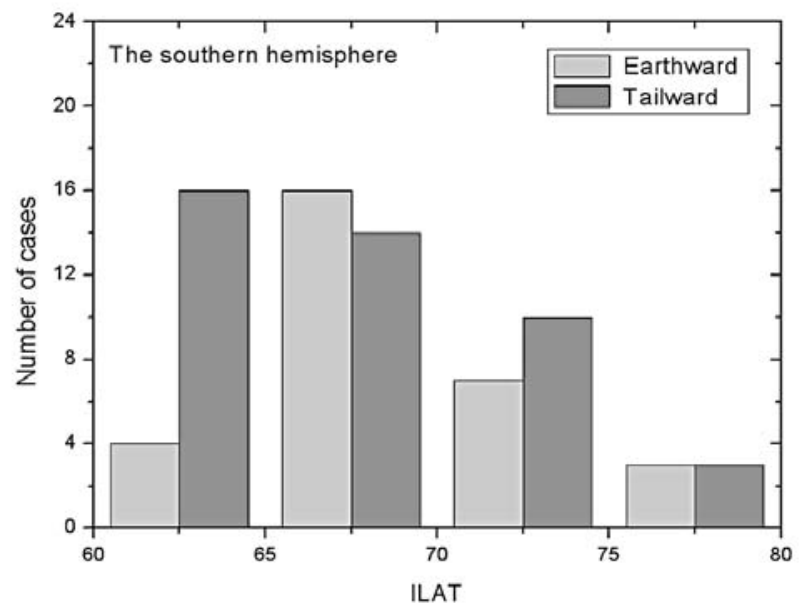

Figure 6. (a) The footprints of the mapping of the FACs along the field line to the polar region: (left) Northern Hemisphere and (right) Southern Hemisphere. The FAC cases in this figure exclude those with the condition $B_{z}<0$. (b) FAC footprint distribution versus invariant latitude (ILAT) in the two hemispheres corresponding to Figure 6a: (left) Northern Hemisphere and (right) Southern Hemisphere. The FAC cases in this figure, of course, exclude those with the condition $B_{z}<0$.

Southern Hemisphere. This shows that the FAC has a wider distribution in longitude in the Southern than in the Northern Hemisphere. Second, the FAC distribution in latitude, in the Southern Hemisphere, is wider before midnight and narrower after midnight compared with those in the Northern Hemisphere (which only has high-latitude cases on the extreme dawnside location). This fact could mean that the southern distribution may contain more cases of reversed polarity arising from the region of the polar cap boundary and therefore could explain the lower dawn-dusk asymmetry in the Southern Hemisphere. Figure $6 \mathrm{~b}$ illustrates the FAC footprint distribution versus latitude in the two hemispheres. We can see that the earthward FAC occurrences in the Northern Hemisphere, in general, are similar to the tailward FACs in each $10^{\circ}$ range from a latitude of $60^{\circ}$ to one of $80^{\circ}$. In the Southern Hemisphere, however, there are differences in the number of earthward and tailward cases at each latitude bin. That is to say, the mapping and the footprints of the FACs are different in the Northern and Southern Hemispheres. The main difference, however, seems to be that the southern distribution is skewed for la- titudes $<65^{\circ}$, with a large, persistent number of tailward FAC cases below $65^{\circ}$. In addition, there is an excess of tailward cases in the latitude range $70^{\circ}-75^{\circ}$.

[19] Closer inspection of Figure 6 shows that the LT distribution of cases reflects that already noted: the tailward cases lie predominantly on the dusk side and the earthward cases lie predominantly on the dawn side in the Northern Hemisphere. We also note that this northern distribution shows no evidence of a large number of reverse boundary currents at high latitudes. The distribution in the Southern Hemisphere, however, shows no clear asymmetry and the tailward and earthward cases are fairly evenly spread in both latitude and LT, with significantly more cases occurring at latitudes higher than $70^{\circ}$ on the dusk side. The low-latitude tailward cases are therefore as much responsible for the reduction in dawn-dusk asymmetry as the higher latitude ones and, therefore, the result does not arise solely from poleward-driven, reverse currents. From the above, we can see that the FAC occurrence, density, and mapping footprints are different in the Northern and Southern Hemispheres. These clearly show that the FACs between the 
ionosphere and magnetosphere have a north-south hemispheric asymmetry.

[20] We would like to mention that a typical thickness of the FAC cases in PSBLs was evaluated as $\sim 4000 \mathrm{~km}$. When we mapped the FACs to the ionospheric height, the thickness comes to $\sim 67 \mathrm{~km}$. This is consistent with the results of other authors [e.g., Frank et al., 1981].

[21] For the dawn-dusk asymmetry of the FACs, we have made further analysis with the attempt to filter out any reconnection-driven currents arising from a NENL located earthward of the spacecraft, i.e., those lying on field lines not connected to the Earth. For the north-south hemispheric asymmetry of the FACs, there are several plausible causes. As we know, the solar wind has control of the FACs in the magnetosphere. Thus, the first cause should be the configuration of the magnetosphere. Because of the geomagnetic dipolar title angle and the solar wind asymmetry related to the magnetosphere, it could result in the FAC north-south hemispheric symmetry in the magnetosphere. The second cause is a change in the ionospheric conductivity [Fujii et al., 1981; Ohtani et al., 2005; Vallat et al., 2005]. Because of the dipole tilt angle, the amount of EUV radiation reaching the ionosphere can change the amount and distribution of conductivity in different hemispheres. As a result, the highlatitude field-aligned intensities can be larger by a factor of $1.5-1.8$ in the sunlit (summer) polar cap in comparison with the winter hemisphere [Christiansen et al., 2002]. This could also result in the north-south hemispheric asymmetry of FACs in the magnetosphere. Christiansen et al. [2002] had offered a different explanation for the FAC system. They argued that the seasonal dependence in the global FAC system is generated and maintained by the various solar wind-magnetosphere interaction processes, such as the quasi-viscous interaction and reconnection. This argument could also be used to interpret the north-south asymmetry. Furthermore, since the current densities of the FACs are different between the Northern and Southern Hemispheres, an important source (but perhaps not the only source) of these currents must be a voltage generator, possibly related to the convective electric field. However, these issues require further investigation and study.

\section{Summary}

[22] We used the data of the fluxgate magnetometer on board the Cluster spacecraft in the period of July to October 2001 and studied the characteristics of the FAC distribution in the plasma sheet boundary layer in the magnetotail. There were 172 FAC cases chosen for the statistics. The current was calculated with the curlometer technique using the 4-point measurement and we took the ratio $|\nabla \cdot \vec{B}| /|\nabla \times \vec{B}|<0.3$ to ensure the data quality. (Typically, we found that the estimates of the FAC density errors are within $12 \%$.) We also attempted to filter out any reconnection-driven currents arising from a NENL located earthward of the spacecraft by removing those cases corresponding to $B_{z}$ negative, and there are 146 FAC cases that have a clear $B_{z}$-positive background field (those most likely to be Earth connected). The main results are as follows.

[23] 1. The FAC distribution on the dawn side is different from that on the dusk side and shows a dusk-dawn asymmetry of earthward and tailward FACs, which is consistent with that expected from the large-scale R1 current system. This is the first time that the dusk-dawn asymmetry of the FACs in the region of $17-19 R_{E}$ in the magnetotail has been confirmed by Cluster observation, otherwise it has been found by the Geotail spacecraft inside $40 R_{E}$ [Ueno et al., 2002].

[24] 2. There is a north-south hemispheric asymmetry of the FACs in the PSBLs: the occurrences of the FACs have different values in the two hemispheres, as do the polarity distributions. In addition, the average density and its standard deviation of the FACs, both for earthward and tailward FACs in the Northern Hemisphere are different from that in the Southern Hemisphere. Furthermore, when we made a mapping of all FACs along the magnetic field lines from the FAC location to the polar ionospheric region, the footprints of the FACs also showed a difference between the Southern and Northern Hemispheres.

[25] We analyzed and discussed the asymmetries of the FACs in the magnetosphere. Asymmetry of the configuration of the magnetosphere and the solar wind, the conductivity in the ionosphere, and the various solar wind-magnetosphere interaction processes (such as the quasi-viscous interaction and reconnection) may be the reasons for the asymmetry of the FACs in the Northern and Southern hemispheres, but it needs further investigation. The fact that the current densities of the FACs are different between the Northern and Southern hemisphere also suggests that an important source (but perhaps not the only source) of these currents is a voltage generator, possibly related to the convective electric field.

[26] Acknowledgments. This research was supported by National Natural Science Foundation of China grants 40874084, 40804031, and 40921063 and by the Specialized Research Fund for State Key Laboratories in China. Portions of this work were carried out as part of the International Space Science Institute working group Comparative Cluster-Double Star Measurements in the Magnetotail and supported by a CAS visiting professorship for senior international scientists. The authors would like to thank the relevant instrument teams for the data to be used in this research.

[27] Thomas Cravens thanks the reviewers for their assistance in evaluating this paper.

\section{References}

Alexeev, I. V., C. J. Owen, A. N. Fazakerley, A. Runov, J. P. Dewhurst, A. Balogh, H. Rème, B. Klecker, and L. Kistler (2005), Cluster observations of currents in the plasma sheet during reconnection, Geophys. Res. Lett., 32, L03101, doi:10.1029/2004GL021420.

Asano, Y., R. Nakamura, W. Baumjohann, A. Runov, A. Voros, M. Volwerk, T. L. Zhang, A. Balogh, B. Kecker, and H. Rème (2005), How typical are atypical current sheets?, Geophys. Res. Lett., 32, L03108, doi:10.1029/ 2004GL021834.

Balogh, A., et al. (2001), The Cluster magnetic field investigation: Overview in flight performance and initial results, Ann. Goephys., 19, 1207-1217.

Christiansen, F., V. O. Papitashvili, and T. Neubert (2002), Seasonal variations of high-latitude field-aligned currents inferred from Ørsted and Magsat observations, J. Geophys. Res., 107(A2), 1029, doi:10.1029/ 2001 JA900104.

Cummings, W. D., and A. J. Dessler (1967), Field-aligned currents in the magnetosphere, J. Geophys. Res., 72(3), doi:10.1029/JZ072i003p01007.

Draper, N. C., et al. (2005), Cluster magnetotail observations of a tailwardtravelling plasmoid at substorm expansion phase onset and field aligned currents in the plasma sheet boundary layer, Ann. Geophys., 23, 36673683 .

Dunlop, M. W., D. J. Southwood, K.-H. Glassmeier, and F. M. Neubauer (1988), Analysis of multipoint magnetometer data, Adv. Space Res., 8, 273. 
Dunlop, M., W. A. Balogh, K.-H. Glassmeier, and P. Robert (2002), Four point Cluster application of magnetic field analysis tools: The curlmeter, J. Geophys. Res., 107(A11), 1384, doi:10.1029/2001JA005088.

Elphic, R. C., P. A. Mutch, and C. T. Russell (1985), Observations of fieldaligned currents at the plasma sheet boundary: An ISEE-1 and 2 survey, Geophys. Res. Lett., 12, 631-634.

Forsyth, C., et al. (2008), Observed tail current systems associated with bursty bulk flows and auroral streamers during a period of multiple substorms, Ann. Geophys., 26, 167-184.

Frank, L. A., R. L. McPherron, R. J. DeCoster, B. G. Burek, K. L. Ackerson, and C. T. Russell (1981), Field-aligned currents in the Earth's magnetotail, J. Geophys. Res., 86, 687-700.

Fujii, R., T. Iijima, T. A. Potemra, and M. Sugiura (1981), Seasonal dependence of large-scale birkeland currents, Geophys. Res. Lett., 8(10), 1105-1106.

Fujimoto, M., T. Nagai, N. Yokokawa, Y. Yamade, T. Mukai, Y. Saito, and S. Kokubun (2001), Tailward electrons at the lobe-plasma sheet interface detected upon dipolarizations, J. Geophys. Res., 106(A10), 21,25521,262, doi:10.1029/2001JA900011.

Iijima, T., and T. A. Potemra (1978), Large-scale characteristics of fieldaligned currents associated with substorms, J. Geophys. Res., 83, 599-615.

Ohtani, S., S. Kokubun, R. C. Elphic, and C. T. Russell (1988), Fieldaligned current signatures in the near-tail region: 1 . ISEE observations in the plasma sheet boundary layer, J. Geophys. Res., 93, 9709-9720.

Ohtani, S., G. Ueno, T. Higuchi, and H. Kawano (2005), Annual and semiannual variations of the location and intensity of large-scale field-aligned currents, J. Geophys. Res., 110, A01216, doi:10.1029/2004JA010634.

Robert, P., and M. W. Dunlop (1998), Accuracy of current density determination, in Analysis Methods for Multi-spacecraft Data, edited by G. Paschmann and D. Patrick, pp. 395-418, Eur. Space Agency, Noordwijk, Netherlands.

Snekvik, K., et al. (2007), Cluster observations of a field aligned current at the dawn flank of a bursty bulk flow, Ann. Geophys., 25, 1405-1415.

Tsyganenko, N. A., and D. P. Stern (1996), Modeling the global magnetic field of the large-scale Birkeland current systems, J. Geophys. Res., 101, 27,187-27,198, doi:10.1029/96JA02735.

Ueno, G., S. Ohtani, Y. Saito, and T. Mukai (2002), Field-aligned currents in the outermost plasma sheet boundary layer with Geotail observation, J. Geophys. Res., 107(A11), 1399 doi:10.1029/2002JA009367.
Vallat, C., I. Dandouras, M. Dunlop, A. Balogh, E. Lucek, G. K. Parks, M. Wilber, E. C. Roelof, G. Chanteur, and H. Rème (2005), First current density measurements in the ring current region using simultaneous multispacecraft Cluster-FGM data, Ann. Geophys., 23, 1849-1865.

Zmuda, A. J., J. H. Martin, and F. T. Heuring (1966), Transverse magnetic disturbance at $1100 \mathrm{~km}$ in the auroral region, J. Geophys. Res., 71 doi:10.1029/JZ071i021p05033.

Z. W. Cheng, Z. X. Liu, C. Shen, and J. K. Shi, State Key Laboratory of Space Weather, Center for Space Science and Applied Research, Chinese Academy of Sciences, PO Box 8701, 100190 Beijing, China. (jkshi@ cssar.ac.cn)

I. Dandouras and H. Rème, Center d'Etude Spatiale des Rayonnements, University of Toulouse, Center National de la Recherche Scientifique, BP 4346, F-31028 Toulouse CEDEX 4, France.

M. Dunlop, Space Science and Technology Department, Rutherford Appleton Laboratory, Didcot OX11 0QX, UK

A. Fazakerley, A. D. Lahiff, and A. P. Walsh, Mullard Space Science Laboratory, University College London, London RH5 6NT, UK.

A. Grocott and M. Lester, Department of Physics and Astronomy, University of Leicester, Leicester LE1 7RH, UK.

L. M. Kistler and C. Mouikis, Department of Physics, University of New Hampshire, 8 College Rd., Morse Hall, Durham, NH 03824-3235, USA.

A. T. Y. Lui, Applied Physics Laboratory, Johns Hopkins University, 11100 Johns Hopkins Rd., Laurel, MD 20723, USA.

E. Lucek, Blackett Laboratory, Space and Atmospheric Physics Group, Imperial College London, South Kensington Campus, London SW7 2AZ, UK.

Z. Y. Pu, School of Earth and Space Sciences, Peking University, 5 Yiheyuan St., 100871 Beijing, China.

M. G. G. T. Taylor, European Space Research and Technology Centre, European Space Agency, Code SCI-SH, Postbus 299, Keplerlaan 1, NL-2200 AG Noordwijk, Netherlands.

K. Torkar, M. Volwerk, and T. L. Zhang, Space Research Institute, Austrian Academy of Sciences, Schmiedlstr. 6, A-8042 Graz, Austria. 